EPJ Web of Conferences 92,02075 (2015)

DOI: $10.1051 /$ epjconf/20159202075

(C) Owned by the authors, published by EDP Sciences, 2015

\title{
Application of Nyström method to a Fredholm integral equation describing induction heating
}

\author{
Josef $\operatorname{Rak}^{1, a}$ \\ ${ }^{1}$ University of Pardubice, Faculty of Electrical Engineering and Informatics, Studentská 9553210 Pardubice \\ Results were obtained by author's doctoral study in Charles University in Prague, Faculty of Mathematics and Physics, \\ Department of Numerical Mathematics

\begin{abstract}
An induction heating problem can be described by a Fredholm Integral Equation of the second kind. The equation is used to compute the eddy current of density. One method for solving such an equation is the Nyström method. It is based on the approximation of the integral in an equation by the numerical integration rule. This paper shows application of the Nyström method to an induction heating problem. Results of the Nyström method are compared with an alternative method.
\end{abstract}

Key words. integral equation of the second kind, induction heating, collocation method, Nyström method

\section{Introduction}

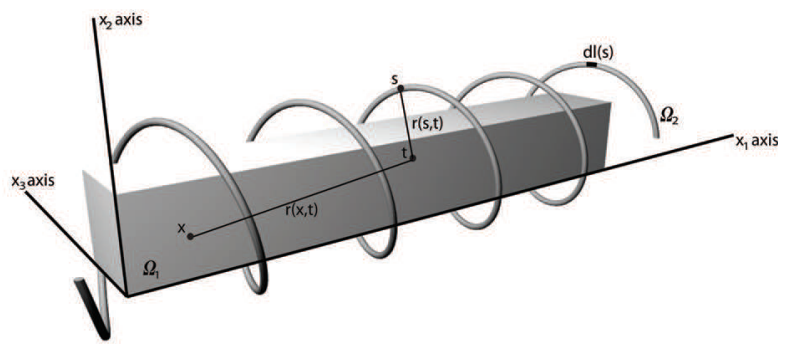

Figure 1. Heated body and coil.

A bounded metal body $\Omega_{1}$ with a Lipschitz-continuous boundary is heated by an external electromagnetic field produced by inductor $\Omega_{2}$ (see figure 1). The inductor is formed by a conductor of general shape and position that carries harmonic current $I_{\text {ext }}$. The main task is to compute the eddy current of density $J_{e d d y}$. In article [8] was derived following equation

$$
\iota J_{e d d y}(x)-\kappa(x) \int_{\Omega_{1}} \frac{J_{e d d y}(t)}{r(x, t)} d t_{1} d t_{2} d t_{3}=\kappa(x) I_{e x t} \int_{\Omega_{2}} \frac{d l(s)}{r(x, s)}
$$

where

$$
\kappa(x)=\frac{\omega \gamma(T(x)) \mu_{0}}{4 \pi},
$$

$r(x, t)$ is the Euclidean distance, $x=\left(x_{1}, x_{2}, x_{3}\right)$ and $t=$ $\left(t_{1}, t_{2}, t_{3}\right)$ are points in the metal body, $s=\left(s_{1}, s_{2}, s_{3}\right)$ is a point at the inductor, $\omega$ is angular frequency, $\gamma$ is electrical conductivity, $\mu_{0}$ is permeability of vacuum and $\iota$ is the complex unit. For each bounded and continuous temperature distribution $T, \kappa(x)$ is a real, positive, bounded

\footnotetext{
a Corresponding author: josef.rak@upce.cz
}

and continuous function. Since $J_{e d d y}$ is a phasor $J_{e d d y}=$ $\left(J_{e d d y, x_{1}}, J_{e d d y, x_{2}}, J_{e d d y, x_{3}}\right)$ we can rewrite (1) into three complex integral equations for $J_{e d d y, x_{1}}, J_{e d d y, x_{2}}$ and $J_{e d d y, x_{3}}$. For the component $J_{e d d y, x_{1}}$ we have

$$
\iota J_{e d d y, x_{1}}(x)-\kappa(x) \int_{\Omega_{1}} \frac{J_{e d d y, x_{1}}(t)}{r(x, t)} d t_{1} d t_{2} d t_{3}=I_{e x t} F(x)
$$

where

$$
F(x)=\kappa(x) \int_{\Omega_{2}} \frac{d l(s) \cdot e_{x_{1}}}{r(x, s)}
$$

where $e_{x_{1}}$ is unite vector $e_{x_{1}}=(1,0,0)$. With the notation $J_{R}(x)=\operatorname{ReJ}_{\text {eddy, } x_{1}}(x), J_{I}(x)=\operatorname{Im}_{\text {eddy, } x_{1}}(x), I_{R}=\operatorname{Re}\left(I_{\text {ext }}\right)$ and $I_{I}=\operatorname{Im}\left(I_{\text {ext }}\right)$ the equation (3) can be split into equivalent system of two real equations

$$
\begin{aligned}
J_{R}(x)-\kappa(x) \int_{\Omega_{1}} \frac{J_{I}(t)}{r(x, t)} d t & =I_{I} F(x), \\
-J_{I}(x)-\kappa(x) \int_{\Omega_{1}} \frac{J_{R}(t)}{r(x, t)} d t & =I_{R} F(x) .
\end{aligned}
$$

Formulas for the remaining components $J_{e d d y, x_{2}}$ and $J_{e d d y, x_{3}}$ can be obtained by mere interchanging of indices. The specific Joule losses which are needed to compute the temperature distribution are given by

$$
\omega_{J}(x)=\frac{1}{\gamma} J_{e}(x) \overline{J_{e}(x)}
$$

where

$$
\begin{gathered}
J_{e}(x)= \\
\sqrt{\left[\operatorname{ReJ}_{e d d y, x_{1}}(x)\right]^{2}+\left[\operatorname{ReJ}_{e d d y, x_{2}}(x)\right]^{2}+\left[\operatorname{ReJ}_{e d d y, x_{3}}(x)\right]^{2}}+ \\
+\iota \sqrt{\left[\operatorname{ImJ}_{e d d y, x_{1}}(x)\right]^{2}+\left[\operatorname{ImJ}_{e d d y, x_{2}}(x)\right]^{2}+\left[\operatorname{ImJ}_{e d d y, x_{3}}(x)\right]^{2}}
\end{gathered}
$$

and $\overline{J_{e}(x)}$ is complex conjugate to $J_{e}(x)$. 


\section{Existence and uniqueness the of solution of the induction heating model}

Equation (3) belongs to the family of Fredholm integral equations of the second kind with the diagonal singular kernel function having the following form

$$
\lambda y(x)-\int_{D} k(x, t) y(t) d t=f(x), x \in D, \lambda \neq 0
$$

where $D \subset R^{m}$ ( $m \geq 1$ ) is assumed to be a closed, bounded and connected set and the kernel function $k(x, t)$ is singular when $x=t$. A set is connected if it is not a subset of a disjoint union of two open sets. Equation (3) describing induction heating problem is of the form (7) with $k(x, t)=$ $\frac{-\iota k(x)}{r(x, t)}, f(x)=-\iota I_{e x t} F(x), y(x)=J_{e d d y, x_{1}}(x), D=\Omega_{1}$ and $\lambda=1$. The operator form of (7) is

$$
(\lambda I-\mathcal{K}) y=f
$$

where integral operator $\mathcal{K}$ is defined by

$$
\mathcal{K} y(x)=\int_{D} k(x, t) y(t) d t
$$

For showing existence and uniqueness of the solution the well known Fredholm alternative theorem is used.

Theorem 1 (Fredholm alternative) Let $\mathcal{X}$ be a Banach space, let $\mathcal{K}: \mathcal{X} \rightarrow \mathcal{X}$ be compact linear operator and let $\lambda \neq 0$. Then either the equation $(\lambda I-\mathcal{K}) y=0$ has $a$ non-trivial solution, or the equation $(\lambda I-\mathcal{K}) y=f$ has a solution for all $f$. In such case the operator $(\lambda I-\mathcal{K})$ has a bounded inverse.

By the last theorem we need to show that operator $\widetilde{\mathcal{K}}$ defined as

$$
\widetilde{\mathcal{K}} y(x)=-\iota \kappa(x) \int_{\Omega_{1}} \frac{y(t)}{r(x, t)} d t
$$

is a compact operator on space $C(D)$ and 1 is not its eigenvalue. The proof of compactness can be done using following lemma. It is a well known result from functional analysis and can be found for example in [1].

Lemma 1 Let $D \subset \mathbb{R}^{n}$ be closed and bounded set. Let the integral operator $\mathcal{K}$ be defined by (9). Finally assume that we can define a sequence of continuous functions $k_{n}(x, t)$ such that it holds

$$
\max _{x \in D} \int_{D}\left|k(x, t)-k_{n}(x, t)\right| d t \rightarrow 0 \text {, as } n \rightarrow \infty .
$$

Then $\mathcal{K}$ is compact linear operator on $C(D)$.

The last lemma cannot be used directly. Let us define operators $\mathcal{K}_{1}, \mathcal{M}_{1}$ and $\mathcal{N}$ from space $C\left(\Omega_{1}\right)$ to space $C\left(\Omega_{1}\right)$ as

$$
\begin{aligned}
\mathcal{K}_{1} y(x) & =\int_{\Omega_{1}} \frac{y(t)}{r(x, t)} d t \\
\mathcal{M}_{1} y(x) & =\kappa(x) y(x) \\
\mathcal{N} y(x) & =\operatorname{Im}(y(x))-\iota \operatorname{Re}(y(x)) .
\end{aligned}
$$

Then for the operator $\widetilde{\mathcal{K}}$ it holds $\widetilde{\mathcal{K}} y(x)=\mathcal{M}_{1} \mathcal{K}_{1} \mathcal{N} y(x)$. Using sphere coordinates and lemma 1 with $k(x, t)=r(x, t)^{-1}$ and $k_{n}(x, t)=r_{n}(x, t)^{-1}$ where

$$
r_{n}(x, t)=\left\{\begin{array}{l}
r_{n}(x, t)=r(x, t), \text { when } r(x, t) \geq \frac{1}{n} \\
r_{n}(x, t)=\frac{1}{n}, \text { when } r(x, t)<\frac{1}{n}
\end{array}\right.
$$

it is simple to prove that $\mathcal{K}_{1}$ is compact operator. Operators $\mathcal{M}$ and $\mathcal{N}$ can be shown to be continuous linear operators. Hence $\widetilde{\mathcal{K}}$ is compact operator. For showing that 1 is not an eigenvalue of $\widetilde{\mathcal{K}}, 1 / \kappa$-weighted inner product can be used and is defined as

$$
(f, g)_{1 / \kappa}=\int_{\Omega_{1}} \frac{f(t) \overline{g(t)}}{\kappa(t)} d t .
$$

Operator $\widetilde{\mathcal{K}}$ can be shown to satisfy

$$
\left(\widetilde{\mathcal{K}} y_{1}, y_{2}\right)_{1 / \kappa}=-\left(y_{1}, \widetilde{\mathcal{K}} y_{2}\right)_{1 / \kappa} \text {, for all } y_{1}, y_{2} \in C\left(\Omega_{1}\right) .
$$

Such an operator is called antisymmetric with respect to the $1 / \kappa$-weighted inner product. The real part of its eigenvalues is equal to 0 , so 1 is not an eigenvalue of operator $\widetilde{\mathcal{K}}$ and by Fredholm alternative is (3) uniquely solvable.

\section{Nyström method}

The Nyström method is based on the approximation of the integral by the numerical integration rule defined as

$$
\int_{D} v(x) d x \approx \sum_{j=1}^{n} \omega_{j} v\left(x_{j}\right)
$$

where $x_{j} \in D$ are called the node points and $\omega_{j}$ weights. Since the kernel function $k(x, t)$ was assumed to be singular when $x=t$, the integration rule cannot be applied directly to (7). The kernel function $k(x, t)$ is approximated by the bounded kernel function $k_{n}(x, t), n=1,2, \ldots$, which coincides with $k(x, t)$ outside a certain neighborhood of $x=t$. Then equation (7) is rewritten into the form as in [6].

$$
\left[\lambda-\int_{D} k(x, t) d t\right] y(x)-\int_{D} k(x, t)[y(t)-y(x)] d t=f(x) .
$$

By changing $k_{n}$ and $k$ in the second integral on the left hand side of (19) and using numerical integration rule we obtain

$$
\begin{gathered}
{\left[\lambda-\int_{D} k(x, t) d t\right] \widetilde{y}_{n}(x)-} \\
-\sum_{j=1}^{n} \omega_{j} k_{n}\left(x, x_{j}\right)\left[\widetilde{y}_{n}\left(x_{j}\right)-\widetilde{y}_{n}(x)\right]=f(x) .
\end{gathered}
$$

Now let us run $x$ through the node points and we get a system of linear equations for $\widetilde{y}_{n}\left(x_{i}\right)$ 


$$
\begin{gathered}
{\left[\lambda+\sum_{\substack{j=1 \\
j \neq i}}^{n} \omega_{j} k_{n}\left(x_{i}, x_{j}\right)-\int_{D} k\left(x_{i}, t\right) d t\right] \widetilde{y}_{n}\left(x_{i}\right)-} \\
\quad-\sum_{\substack{j=1 \\
j \neq i}}^{n} \omega_{j} k_{n}\left(x_{i}, x_{j}\right) \widetilde{y}_{n}\left(x_{j}\right)=f\left(x_{i}\right) .
\end{gathered}
$$

The numerical solution is obtained by the interpolation formula

$$
\widetilde{y}_{n}(x)=\frac{f(x)+\sum_{j=1}^{n} \omega_{j} k_{n}\left(x, x_{j}\right) \widetilde{y}_{n}\left(x_{j}\right)}{\lambda+\sum_{j=1}^{n} \omega_{j} k_{n}\left(x, x_{j}\right)-\int_{D} k(x, t) d t} .
$$

The integral can be calculated analytically or by some special numerical integration rule.

The problem is to define the bounded approximation $k_{n}(x, t)$. To do it, we need more assumptions to the original kernel function $k(x, t)$. Let $r(x, t)$ be the Euclidean distance of points $x, t \in D$. Let

$$
R_{D}=\max _{x, t \in D} r(x, t)
$$

Assume that there exists function $h \in C(D \times D)$ and the positive non-increasing function $g \in C(0, \infty)$ satisfying

$$
\lim _{t \rightarrow 0+} g(t)=\infty,
$$

such that the kernel function is of the form

$$
k(x, t)=g(r(x, t)) h(x, t) .
$$

Now let us define bounded kernel approximation $k_{n}(x, t)$. Assume that $\mu_{n}$ is a decreasing positive sequence such that

$$
\lim _{n \rightarrow \infty} \mu_{n}=0 .
$$

Then kernel function can be approximated by function

$$
k_{n}(x, t)=g_{\mu_{n}}(r(x, t)) h(x, t),
$$

where

$$
g_{\mu_{n}}(u)=\left\{\begin{array}{l}
g(u), \text { if } u \geq \mu_{n} \\
g\left(\mu_{n}\right), \text { if } u<\mu_{n}
\end{array}\right.
$$

Since $g \in C(0, \infty)$ was a positive non-increasing function, $g_{\mu_{n}} \in C[0, \infty)$, is also a positive and non-increasing function for all $n$. Note that approximation (26) is a bounded approximation of the original $k(x, t)$.

For the proof of convergence are needed further assumptions to the kernel function and numerical integration rule. Assume that the numerical integration rule is convergent for all continuous functions and all its weights are positive. Let us define

$$
\bar{\omega}_{n}=\max _{j=1, \ldots, n} \omega_{j}
$$

For the sequence $\mu_{n}$ assume that

$$
\mu_{n}^{m} \geq \rho^{m} \bar{\omega}_{n},
$$

where $0<\rho<\infty$ and that there exists $\bar{\mu}<\infty$ such that

$$
g\left(\mu_{n}\right) \bar{\omega}_{n} \leq \bar{\mu} \text { for all } n .
$$

Also assume that there exists $c_{D}<\infty$ such that

$$
\max _{x \in D} \int_{\left\{t, r(x, t)<R_{D}\right\}} g(r(x, t)) d t \leq c_{D}
$$

and

$$
\lim _{v \rightarrow 0} \max _{x \in D} \int_{\{t, r(x, t)<v\}} g(r(x, t)) d t=0 .
$$

Finally assume that there exists constant $c<\infty$ such that for all positive, non-increasing function $z \in C[0, \infty)$ and $x \in D$ holds following inequality

$$
\sum_{j, r\left(x, x_{j}\right) \leq \xi} \omega_{j} z\left(r\left(x, x_{j}\right)\right) \leq c\left[z(0) \bar{\omega}_{n}+\int_{\{t, r(x, t) \leq \xi\}} z(r(x, t)) d t\right] .
$$

The last conditions seem to be very restrictive, however with some effort it can be proven that it is satisfied for several compound numerical integration rules (for example if $D$ is a rectangle or cuboid then it can be proved to be satisfied for the compound mid-rectanglular or the compound mid-cuboid rule). The convergence is done by operator calculus. Specifically the by following theorem. It is a modified version of theorem 4.1.1 from [3].

Theorem 2 Let $\mathcal{X}$ be a Banach space, let operators $\mathcal{S}, \mathcal{T}$ be bounded on $\mathcal{X}$. For given $\lambda \neq 0$ let us assume that $\lambda I-$ $\mathcal{T}$ is a bijection on $\mathcal{X}$ (which means $(\lambda I-\mathcal{T})^{-1}$ exists, is bounded and $\mathcal{R}(\lambda I-\mathcal{T})=\mathcal{X})$. If

$$
\|(\mathcal{T}-\mathcal{S}) \mathcal{S}\|<\frac{|\lambda|}{\left\|(\lambda I-\mathcal{T})^{-1}\right\|}
$$

then $(\lambda I-\mathcal{S})^{-1}: \mathcal{R}(\lambda I-\mathcal{S}) \rightarrow \mathcal{X}$ exists, is bounded and

$$
\left\|(\lambda \mathcal{I}-\mathcal{S})^{-1}\right\| \leq \frac{1+\left\|(\lambda I-\mathcal{T})^{-1}\right\|\|\mathcal{S}\|}{|\lambda|-\left\|(\lambda I-\mathcal{T})^{-1}|\|\mid(\mathcal{T}-\mathcal{S}) \mathcal{S}\|\right.} .
$$

Let $f \in \mathcal{R}(\lambda I-\mathcal{S})$. Let $y$ be solution of $(\lambda I-\mathcal{T}) y=f$ and let $z$ be solution of $(\lambda I-\mathcal{S}) z=f$. Then it holds

$$
\|y-z\|_{\mathcal{X}} \leq\left\|(\lambda I-\mathcal{S})^{-1}\right\|\left\|\mathcal{T} y-\mathcal{S}_{y}\right\|_{\mathcal{X}} .
$$

If $S$ is compact operator then $\mathcal{R}(\lambda I-\mathcal{S})=\mathcal{X}$.

First equation (20) needs to be rewritten into operator form. With the definition

$$
\begin{aligned}
\mathcal{K}_{n} y(x) & =\sum_{j=1}^{n} \omega_{j} k_{n}\left(x, x_{j}\right) y\left(x_{j}\right) \\
\widetilde{\mathcal{K}}_{n} y(x) & =\sum_{j=1}^{n} \omega_{j} k_{n}\left(x, x_{j}\right)\left[y\left(x_{j}\right)-y(x)\right]+ \\
& +\int_{D} k(x, t) y(x) d t .
\end{aligned}
$$

(20) is equivalent to

$$
\left(\lambda I-\widetilde{\mathcal{K}}_{n}\right) \widetilde{y}_{n}=f .
$$

We need to verify condition (34) for $\mathcal{T}=\mathcal{K}$ and $\mathcal{S}=$ $\widetilde{\mathcal{K}}_{n}$. It can be done with the theory of collectively compact operators described by Anselone in [5]. 
Definition 1 Let $\mathcal{X}$ be a Banach space. Let $\left\{\mathcal{K}_{n}, n \geq 1\right\}$ be a family of linear operators on $\mathcal{X}$ into $\mathcal{X}$. Assume that the set

$$
\left\{\mathcal{K}_{n} y, n \geq 1,\|y\| \leq 1\right\}
$$

has compact closure in $\mathcal{X}$. Then $\left\{\mathcal{K}_{n}, n \geq 1\right\}$ is called collectively compact family of operators on $\mathcal{X}$.

The most important properties of collectively compact operators related to integral equations of the second kind are summarized in following theorem

Theorem 3 Let $\mathcal{X}$ be a Banach space and let $\mathcal{K}$ be a linear operator defined on $\mathcal{X}$ into $\mathcal{X}$. Assume that $\left\{\mathcal{K}_{n}, n \geq 1\right\}$ is collectively compact family of operators on $\mathcal{X}$. Finally assume that

$$
\mathcal{K}_{n} y \rightarrow \mathcal{K} \text { y as } n \rightarrow \infty \text { for all } y \in \mathcal{X}
$$

Then $\mathcal{K}$ is compact operator, for any compact operator $\mathcal{M}: \mathcal{X} \rightarrow \mathcal{X}$ it holds

$$
\left\|\left(\mathcal{K}-\mathcal{K}_{n}\right) \mathcal{M}\right\| \rightarrow 0 \text { as } n \rightarrow \infty
$$

and

$$
\left\|\left(\mathcal{K}-\mathcal{K}_{n}\right) \mathcal{K}_{n}\right\| \rightarrow 0 \text { as } n \rightarrow \infty .
$$

Assume that the kernel function is of the form (24), where $g$ satisfies (23). Also assume that the numerical integration rule converges for all continuous functions and has positive weights. Finally assume that (29) - (33) hold. Then the sequence of operators $\mathcal{K}_{n}$ is collectively compact approximation of $\mathcal{K}$. Since operator $\tilde{\mathcal{K}}_{n}$ can be written as

$$
\widetilde{\mathcal{K}}_{n}=\mathcal{K}_{n}-\left(\mathcal{K}_{n} u-\mathcal{K} u\right) \mathcal{I}
$$

where $u(x)=1$ and $\mathcal{I}$ is an identical operator, there is no problem to show that from (42) follows that

$$
\begin{aligned}
\left\|\left(\mathcal{K}-\widetilde{\mathcal{K}}_{n}\right) \widetilde{\mathcal{K}}_{n}\right\| & \rightarrow 0 \\
\left\|\mathcal{K}_{n}-\widetilde{\mathcal{K}}_{n}\right\| & \rightarrow 0 .
\end{aligned}
$$

However operator $\widetilde{\mathcal{K}}_{n}$ is not compact. From (36) in theorem 2 we get for $\mathcal{T}=\mathcal{K}$ and $\mathcal{S}=\widetilde{\mathcal{K}}_{n}$

$$
\left\|y-\widetilde{y}_{n}\right\|_{\infty} \leq\left\|( \lambda I - \widetilde { \mathcal { K } } _ { n } ) ^ { - 1 } \left|\left\|\mid \mathcal{K} y-\widetilde{\mathcal{K}}_{n} y\right\|_{\infty} .\right.\right.
$$

But the existence of $\widetilde{y}_{n}$ is guaranteed only for $f \in \mathcal{R}(\lambda I-$ $\widetilde{\mathcal{K}}_{n}$ ). However we have the following identity

$$
\lambda \mathcal{I}-\widetilde{\mathcal{K}}_{n}=\left[\mathcal{I}-\mathcal{K}_{n}\left(\lambda I-\widetilde{\mathcal{K}}_{n}+\mathcal{K}_{n}\right)^{-1}\right]\left(\lambda \mathcal{I}-\widetilde{\mathcal{K}}_{n}+\mathcal{K}_{n}\right)
$$

and well known proposition from functional analysis.

Proposition 1 (Inverse Theorem proposition) Let $\mathcal{X}$ be a Banach space and let $\mathcal{T}: \mathcal{X} \rightarrow \mathcal{X}$ be continuous linear operator. Assume that

$$
\frac{1}{|\lambda|}\|\mathcal{T}\|<1
$$

Then $(\lambda I-\mathcal{T})$ has inverse, which means that $(\lambda I-\mathcal{T})^{-1}$ : $\mathcal{X} \rightarrow \mathcal{X}$ is a bounded linear operator.
From (44) and proposition 1 we have that $\left(\lambda I-\widetilde{\mathcal{K}}_{n}+\right.$ $\left.\mathcal{K}_{n}\right)^{-1}$ exists as a bijection from $C(D)$ to $C(D)$ for all sufficiently large $n$. Since the left hand side of (46) is invertible then the right hand side is also invertible. So the operator $\left.\mathcal{I}-\mathcal{K}_{n}\left(\lambda \mathcal{I}-\widetilde{\mathcal{K}}_{n}+\mathcal{K}_{n}\right)^{-1}\right]$ must be one-to-one, otherwise the right hand side of (46) would not be invertible. Since $\mathcal{K}_{n}\left(\lambda I-\widetilde{\mathcal{K}}_{n}+\mathcal{K}_{n}\right)^{-1}$ is a compact operator it follows from the Fredholm alternative theorem 1 that the operator $\left[\mathcal{I}-\mathcal{K}_{n}\left(\lambda \mathcal{I}-\widetilde{\mathcal{K}}_{n}+\mathcal{K}_{n}\right)^{-1}\right]^{-1}$ exists from $C(D)$ into $C(D)$. From this and (46) we get

$$
\begin{gathered}
\left(\lambda I-\widetilde{\mathcal{K}}_{n}\right)^{-1}= \\
\left(\lambda I-\widetilde{\mathcal{K}}_{n}+\mathcal{K}_{n}\right)^{-1}\left[\mathcal{I}-\mathcal{K}_{n}\left(\lambda \mathcal{I}-\widetilde{\mathcal{K}}_{n}+\mathcal{K}_{n}\right)^{-1}\right]^{-1} .
\end{gathered}
$$

Both operators on the right hand side of (48) exist as operators from $C(D)$ to $C(D)$. Hence

$$
\begin{gathered}
\mathcal{R}\left(\lambda I-\widetilde{\mathcal{K}}_{n}\right)= \\
=\mathcal{R}\left[\mathcal{I}-\mathcal{K}_{n}\left(\lambda I-\widetilde{\mathcal{K}}_{n}+\mathcal{K}_{n}\right)^{-1}\right]\left(\lambda I-\widetilde{\mathcal{K}}_{n}+\mathcal{K}_{n}\right)=C(D)
\end{gathered}
$$

and operator $\left(\lambda I-\widetilde{\mathcal{K}}_{n}\right)^{-1}$ exists as an operator from $C(D)$ to $C(D)$ and we get the existence of a numerical solution and convergence of the Nyström method.

\subsection{Application of Nyström method}

For simplicity let us assume that the metal body $\Omega_{1}$ is cuboid. Let us use the compound mid-cuboid rule. Let $x_{i}$ be the node points defined as center of sub-cuboides. For the weight let

$$
\omega_{j}=\omega=\frac{\left|\Omega_{1}\right|}{n}, j=1, \ldots, n .
$$

Let $r_{n}(x, t)$ be approximation of $r(x, t)$ which coincide outside a certain neighborhood of $x=t$. The exact construction is immaterial. From (21) we get a system of linear equations for $\widetilde{J}_{n}\left(x_{i}\right)$

$$
\begin{aligned}
& {\left[1-\iota \kappa\left(x_{i}\right) \sum_{\substack{j=1 \\
j \neq i}}^{n} \frac{\omega}{r_{n}\left(x_{i}, x_{j}\right)}++\iota \kappa\left(x_{i}\right) \int_{\Omega_{1}} \frac{d t_{1} d t_{2} d t_{3}}{r\left(x_{i}, t\right)}\right] \widetilde{J}_{n}\left(x_{i}\right)+} \\
& \quad+\iota \kappa\left(x_{i}\right) \sum_{\substack{j=1 \\
j \neq i}}^{n} \frac{\omega \widetilde{J}_{n}\left(x_{j}\right)}{r_{n}\left(x_{i}, x_{j}\right)}=-\iota I_{e x t} F\left(x_{i}\right), i=1, \ldots, n
\end{aligned}
$$

$\widetilde{J}_{n}\left(x_{i}\right)$ is complex number. Let us define for each $i=1, \ldots, n$

$$
\widetilde{J}_{i}^{(R)}=\operatorname{Re} \widetilde{J}_{n}\left(x_{i}\right) \text { and } \widetilde{J}_{i}^{(I)}=\operatorname{Im} \widetilde{J}_{n}\left(x_{i}\right) .
$$

Then (49) is equivalent the system

$$
\begin{gathered}
\widetilde{J}_{i}^{(R)}+\kappa\left(x_{i}\right)\left[\sum_{\substack{j=1 \\
j \neq i}}^{n} \frac{\omega}{r_{n}\left(x_{i}, x_{j}\right)}-\int_{\Omega_{1}} \frac{d t_{1} d t_{2} d t_{3}}{r\left(x_{i}, t\right)}\right] \widetilde{J}_{i}^{(I)}- \\
-\kappa\left(x_{i}\right) \sum_{\substack{j=1 \\
j \neq i}}^{n} \frac{\omega \widetilde{J}_{j}^{(I)}}{r_{n}\left(x_{i}, x_{j}\right)}=\operatorname{Im} I_{e x t} F\left(x_{i}\right), i=1, \ldots, n
\end{gathered}
$$




$$
\begin{aligned}
& -\widetilde{J}_{i}^{(I)}+\kappa\left(x_{i}\right)\left[\sum_{\substack{j=1 \\
j \neq i}}^{n} \frac{\omega}{r_{n}\left(x_{i}, x_{j}\right)}-\int_{\Omega_{1}} \frac{d t_{1} d t_{2} d t_{3}}{r\left(x_{i}, t\right)}\right] \widetilde{J}_{i}^{(R)}- \\
& -\kappa\left(x_{i}\right) \sum_{\substack{j=1 \\
j \neq i}}^{n} \frac{\omega \widetilde{J}_{j}^{(R)}}{r_{n}\left(x_{i}, x_{j}\right)}=\operatorname{Re} I_{e x t} F\left(x_{i}\right), i=1, \ldots, n
\end{aligned}
$$

Following Nyström interpolation formula (22) we get the numerical solution for eddy currents $\widetilde{J}_{n}$

$$
\widetilde{J}_{n}(x)=\frac{-\iota I_{e x t} F(x)-\iota \kappa(x) \sum_{j=1}^{n} \frac{\omega}{r_{n}\left(x, x_{j}\right)} \widetilde{J}_{n}\left(x_{j}\right)}{1-\iota \kappa(x) \sum_{j=1}^{n} \frac{\omega}{r_{n}\left(x, x_{j}\right)}+\iota \kappa(x) \int_{\Omega_{1}} \frac{1}{r(x, t)} d t},
$$

where

$$
\widetilde{J}_{n}\left(x_{i}\right)=\widetilde{J}_{i}^{(R)}+\iota \widetilde{J}_{i}^{(I)} .
$$

Since since $\Omega_{1}$ is cuboid the integral in (51) and (52) can be computed analytically.

\section{Collocation method}

To compare the results of Nyström method we will use the piecewise constant collocation method described in [7]. Let us cover $\Omega_{1}$ by a regular mesh of $n$ sub-cuboides $\Omega_{1,1}, . ., \Omega_{1, n}$ and let the approximation points be the center points of sub-cuboides. Applying the piecewise constant collocation we get a numerical solution

$$
J_{n}(x)=\sum_{i=1}^{n} \chi_{i}(x) J_{i}
$$

where $\chi_{i}=\chi_{\Omega_{1, i}}$ is a characteristic function of $\Omega_{1, i}$ and $J_{i}$ is the solution of a system of linear equations

$$
\begin{gathered}
J_{i}+\iota \sum_{j=1}^{n} \kappa\left(x_{i}\right) J_{j} \int_{\Omega_{1, j}} \frac{1}{r\left(x_{i}, t\right)} d t_{1} d t_{2} d t_{3}= \\
=-\iota I_{\text {ext }} F\left(x_{i}\right), i=1, \ldots, n .
\end{gathered}
$$

Let us define for each $i=1, \ldots, n J_{i}^{(R)}=\operatorname{Re} J_{i}$ and $J_{i}^{(I)}=$ $\operatorname{Im} J_{i}$. Then the system of linear equations (54) can be rewritten into system of linear equations for $J_{i}^{(R)}$ and $J_{i}^{(I)}$ :

$$
\begin{aligned}
& J_{i}^{(R)}-\sum_{j=1}^{n} \kappa\left(x_{i}\right) J_{j}^{(I)} \int_{\Omega_{1, j}} \frac{d t_{1} d t_{2} d t_{3}}{r\left(x_{i}, t\right)}=\operatorname{Im} I_{e x t} F\left(x_{i}\right), i=1, \ldots, n \\
& -J_{i}^{(I)}-\sum_{j=1}^{n} \kappa\left(x_{i}\right) J_{j}^{(R)} \int_{\Omega_{1, j}} \frac{d t_{1} d t_{2} d t_{3}}{r\left(x_{i}, t\right)}=\operatorname{ReI}_{e x t} F\left(x_{i}\right), i=1, \ldots, n .
\end{aligned}
$$

The numerical solution is (53) with $J_{i}=J_{i}^{(R)}+\iota J_{i}^{(I)}$. The singular integral in the system of linear equations can be also calculated analytically here.

\section{Example}

A brass cuboid body with the size $0.15 \times 0.01 \times 0.01 \mathrm{~m}$ (see figure 2) is heated with a stationary inductor starting at the room temperature $20{ }^{\circ} \mathrm{C}$. The inductor has the form of a coil which turns around the heated body in the $x_{1}$-direction in 6 loops. The radius of the coil is $0.015 \mathrm{~m}$, exciting current $500 \mathrm{~A}$ and frequency $150 \mathrm{kHz}$. The length of the coil is $0.15 \mathrm{~m}$.

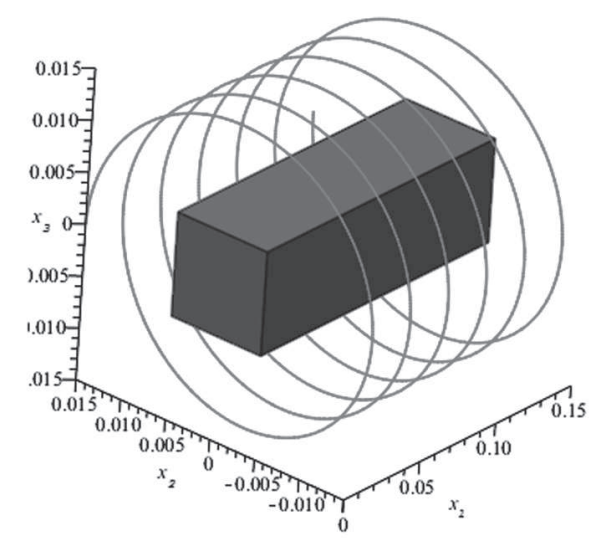

Figure 2. Heating of a brass body - 6 loops

The cuboid is partitioned by 75 elements in $x_{1}$ direction, 5 elements in $x_{2}$ and $x_{3}$ direction. Figure 3 shows the specific Joule losses distribution calculated by the piecewise constant collocation and the Nyström method with the compound mid-cuboid integration rule on the $x_{1}$ axes with $x_{2}=-0.004$ and $x_{3}=-0.004$. The same situation with $x_{3}=0$ is shown in figure 4 .

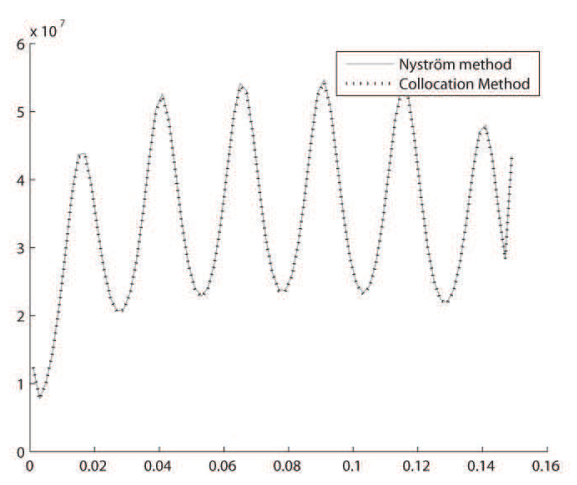

Figure 3. 6 loops, $x_{2}=-0.004, x_{3}=-0.004$

Computation was made by Matlab. The integration for computing $F\left(x_{i}\right)$ is done by the Matlab's method quad. It uses adaptive the Simpson quadrature. For the computation of non-singular integrals of the matrix of collocation method $\operatorname{simp} 3 D$ is used. It was developed by W. Padden, Ch. Macaskill from The University of Sydney in 2008. It uses compound product Simpson integration rule. Matlab has the tool triplequad for computing triple integrals, but it is too slow.

To see the dependence of Joule loses and coil structure let us make another example with one change. The coil 


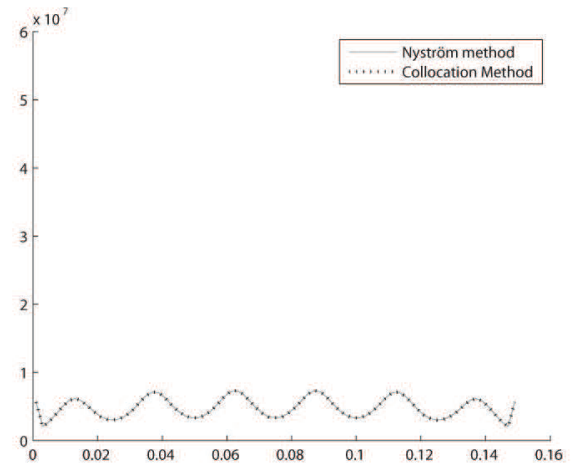

Figure 4. 6 loops, $x_{2}=-0.004, x_{3}=0$

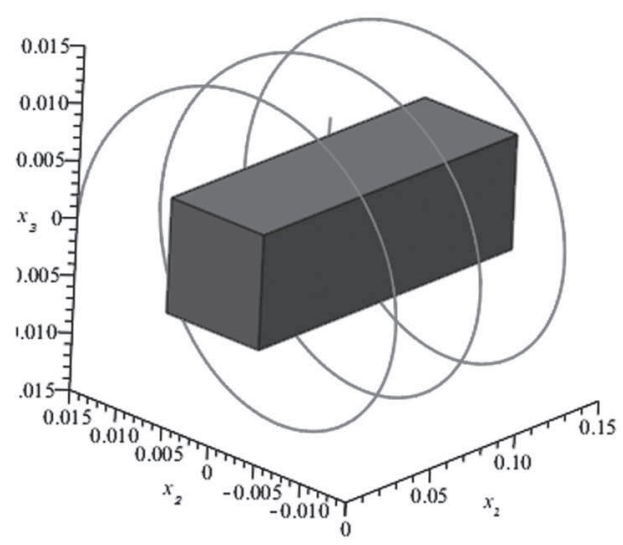

Figure 5. Heating of a brass body - 3 loops

turns around the heated body in the $x_{1}$-direction in 3 loops (see figure 5). Figure 6 shows the specific Joule losses distribution calculated the by piecewise constant collocation and the Nyström method with the compound midcuboid integration rule on the $x_{1}$ axes with $x_{2}=-0.004$ and $x_{3}=-0.004$. The same situation with $x_{3}=0$ is shown in figure 7.

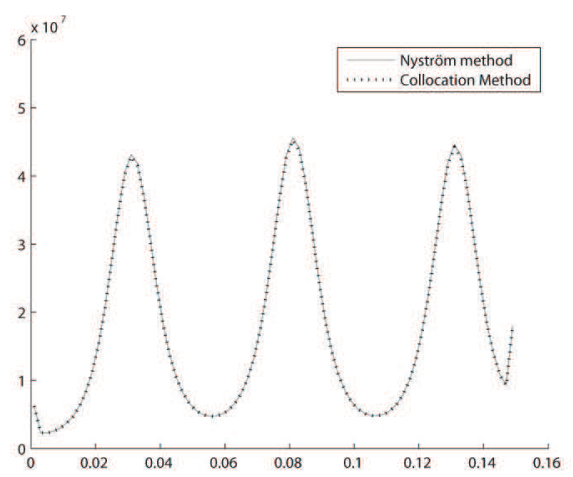

Figure 6. 3 loops, $x_{2}=-0.004, x_{3}=-0.004$

\section{Conclusion}

From the pictures we can see that both methods have similar results. Also the dependence on distance of the point

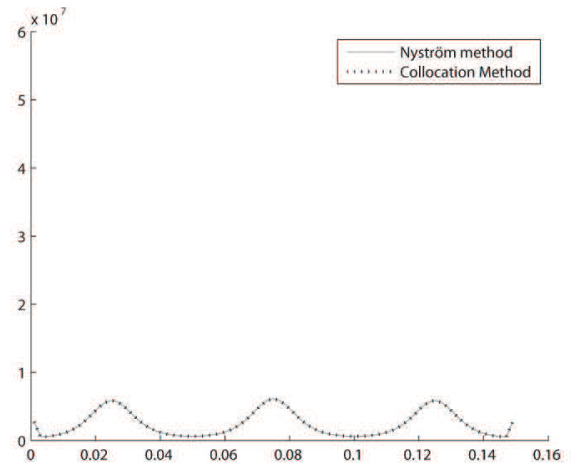

Figure 7. 3 loops, $x_{2}=-0.004, x_{3}=0$

in the body from the coil is well described by the pictures above. If we compare computing time on operating system Windows 7 with Inter Core i5, 4GB ram we get approximately 2 minutes for the Nyström method and approximately 12 minutes for piecewise constant collocation. This is main and big advantage of the Nyström method. It reduces time needed for computation.

\section{References}

1. J. Lukes, Zapisky z funkcionalni analyzy (Karolinum, Praha, 1998)

2. J. Lukes, J. Maly, Mira a Integral (Karolinum, Praha, 1993)

3. K. E. Atkinson, The Numerical Solution of Integral Equations of the Second Kind (Cambridge University Press, 1997)

4. K. E. Atkinson, W. Han, Theoretical Numerical Analysis (Springer-Verlag, New York, 2001)

5. P. M. Anselone, Collectively compact operator approximation theory and applications to integral equations (Prentice-Hall, Inc., Englewood Cliffs, N.J. 1971)

6. L. V. Kantorovich, V. I. Krylov, Approximate Methods of Higher Analysis, (Interscience New York, 1958)

7. K. Atkinson, I. Graham and I. Sloan, SIAM J. Numer. Anal. 20, 172-186 (1983)

8. P. Solin, I. Dolezel, M. Skopek, B. Ulrych, SPETO 2001 International Conference, 143-146 (2001) 